\title{
Internal jugular vein thrombosis associated with Granulicatella adiacens
}

\author{
Honami Kawai, Toshiaki Shiojiri
}

Department of General Internal Medicine, Kokuho Asahi Chuo Hospital, Asahi, Chiba, Japan

Correspondence to Dr Honami Kawai; h.kawai8016@gmail.com

Accepted 29 December 2020

\section{SUMMARY}

Granulicatella adiacens, which occurs as part of the oral microflora, is an uncommon cause of infection. However, it can cause serious bloodstream infections including infective endocarditis. Although oral bacteria, most commonly the Fusobacterium spp, can cause internal jugular vein (IJV) thrombophlebitis, there are no reported cases of IJV thrombosis caused by $G$. adiacens. Here we report a patient with septic IJV thrombosis with $G$. adiacens bacteraemia. A middle-aged man presented to our hospital with fever and altered mental status. Blood cultures were positive for $G$. adiacens, and pan-scan CT with contrast showed left IJV thrombosis, pulmonary embolism and abscesses in the gluteal muscles. The patient was successfully treated with antibiotics. When confronted with $G$. adiacens bacteraemia in patients with poor oral hygiene, it is necessary to be cautious of the fact that this organism can cause IJV thrombophlebitis.

\section{BACKGROUND}

Although previous reports describe Fusobacterium spp as the most common organism responsible for internal jugular vein (IJV) thrombophlebitis, there are some reports indicating other oral bacteria. Granulicatella adiacens is part of the normal oral microflora and is an uncommon clinical isolate which can cause serious bloodstream infections. This is the first reported case of IJV thrombosis associated with $G$. adiacens.

\section{CASE PRESENTATION}

A 58-year-old Japanese man was found unconscious on the floor of his home and brought to our hospital by emergency medical services. His medical history was significant for bipolar disorder treated with lithium. He had no history of diabetes, immunological or haematological disorders. No prosthesis was found in his body. There were no records of recent dental procedures or intra-articular injections. On arrival the patient appeared ill and febrile with a temperature of $40.4^{\circ} \mathrm{C}$, tachypnoeic with a respiratory rate of 26 respirations/min and his oxygen saturation was $98 \%$ on breathing room air. His blood pressure was $138 / 97 \mathrm{mmHg}$ and he had a pulse of $98 \mathrm{bpm}$. The Glasgow Coma Scale score was E4V1M1 without any neck stiffness or focal neurological deficits. He was thin with a height of $173 \mathrm{~cm}$ and body weight of $54 \mathrm{~kg}$. Although he had oral contamination, no signs of pharyngitis or tonsillitis were observed, and no swelling or erythema was detected on his neck. No murmurs were audible on auscultation. We also did not detect any petechiae, splinter haemorrhages, Osler's nodes or Janeway lesions. Initial blood tests revealed increased inflammatory markers with C-reactive protein of $2.42 \mathrm{mg} / \mathrm{dL}$, leukocytosis with white blood cell count of $21500 / \mu \mathrm{L}$ and platelet count of $26.4 \times 10^{4} / \mu \mathrm{L}$. Impaired renal function with uraemia was detected with urea levels of $60 \mathrm{mg}$ / $\mathrm{dL}$, creatinine levels of $2.21 \mathrm{mg} / \mathrm{dL}$ and an elevated lithium level of $2.08 \mathrm{mEq} / \mathrm{L} \quad(0.6-1.2 \mathrm{mEq} / \mathrm{L})$. The blood glucose level was $10.4 \mathrm{mmol} / \mathrm{L}$ and the lactate level was $2.53 \mathrm{mmol} / \mathrm{L}$ (table 1). Head CT/ MRI revealed no significant lesions, and analysis of cerebrospinal fluid (CSF) was unremarkable. The patient was diagnosed with lithium intoxication and underwent haemodialysis. Blood culture samples were taken on admission of the patient, taking into consideration septic encephalopathy as one of the differentials. They were positive for Gram positive cocci (GPC) chains after 20 hours of incubation. On day 3, the GPC chains from the blood culture were identified as $G$. adiacens which was sensitive to ampicillin or cefotaxime.

\section{INVESTIGATIONS}

To investigate the entry and focus of the infection, a pan-scan CT with contrast was performed revealing left cervical vein thrombosis (figure 1), multiple intramuscular abscesses around the trochanteric region of the left femur (figure 2) and pulmonary embolism (figure 3). No abscesses were observed around the oropharynx region. Dental examination revealed poor dental hygiene with periodontitis. The patient had no other focal infection sites in his oral cavity. A transthoracic echocardiogram showed no vegetation or valvular dysfunction. CSF culture and viral PCR were all negative.

\section{DIFFERENTIAL DIAGNOSIS}

We suspected that $G$. adiacens infection in the mouth had spread to the IJV by the haematogenous route, with septic metastases in the lung and gluteal muscles. Although physical examination showed no signs of classical peripheral endocarditis and the results of the transthoracic echocardiogram were unrevealing, the possibility of endocarditis remained. However, we did not perform transoesophageal echocardiography to determine endocarditis as it did not affect the prognosis of this case, which was long-term antibiotic management until the resolution of the abscesses and thrombophlebitis.

\section{TREATMENT}

The patient was treated with ampicillin and subsequent blood culture samples collected on day 3 were 


\begin{tabular}{lll}
\hline Table 1 & Laboratory results at presentation & \\
\hline Test & Result & Reference range \\
White cell count & $21500 / \mu \mathrm{L}$ & $3300-8600 / \mu \mathrm{L}$ \\
\hline Haemoglobin & $14.9 \mathrm{~g} / \mathrm{dL}$ & $13.5-16.9 \mathrm{~g} / \mathrm{dL}$ \\
\hline Platelets & $26.4 \times 10^{4} / \mu \mathrm{L}$ & $15.8-35.310^{4} / \mu \mathrm{L}$ \\
\hline C-reactive protein & $2.42 \mathrm{mg} / \mathrm{dL}$ & $<0.15 \mathrm{mg} / \mathrm{dL}$ \\
\hline Urea & $60 \mathrm{mg} / \mathrm{dL}$ & $8-20 \mathrm{mg} / \mathrm{dL}$ \\
Creatinine & $2.21 \mathrm{mg} / \mathrm{dL}$ & $0.65-1.06 \mathrm{mg} / \mathrm{dL}$ \\
\hline Lithium & $2.08 \mathrm{mEq} / \mathrm{L}$ & $0.6-1.2 \mathrm{mEq} / \mathrm{L}$ \\
\hline Plasma glucose & $10.4 \mathrm{mmol} / \mathrm{L}$ & $3.9-5.6 \mathrm{mmo} / \mathrm{L}$ \\
Serum lactate & $2.53 \mathrm{mmol} / \mathrm{L}$ & $0.44-1.78 \mathrm{mmol} / \mathrm{L}$ \\
\hline
\end{tabular}

negative. On day 16 we changed the antibiotics from ampicillin to cefotaxime as he was suspected to have a drug fever caused by the ampicillin. Following a CT scan on day 40 , we observed that the pulmonary embolism had gone away. However, the left cervical vein thrombus had not diminished in size, and abscesses around his left femur had become slightly larger, requiring incision and drainage. A culture test of the drained pus was unrevealing. After drainage, edoxaban therapy was commenced. The CT scan on day 56 showed that the left cervical vein thrombus and gluteal abscesses had resolved. Erythrocyte sedimentation rate (ESR) levels were also normalised and antibiotics were switched from intravenous cefotaxime to oral amoxicillin on day 70. However, areas of restricted drug diffusion around the trochanteric region of the left femur were still visible on MRI at day 90. We consulted radiologists and microbiologists, and they prescribed continuation of antibiotics because the gluteal abscesses did not seem to have disappeared completely.

\section{OUTCOME AND FOLLOW-UP}

He was discharged on day 98 with oral amoxicillin for an additional 2 weeks and long-term administration of edoxaban, and transferred to another hospital to receive extended psychiatric care.

\section{DISCUSSION}

Jugular vein thrombophlebitis is characterised by infection of the carotid sheath vessels caused by bacteraemia. The causative organisms of this condition are usually members of the normal oropharyngeal flora with Fusobacterium necrophorum being the most common pathogen. It is also known as Lemierre's syndrome when it is caused by Fusobacterium spp. ${ }^{1}$ This condition is primarily preceded by head and neck infections such as pharyngitis and primary dental infection. IJV emerges as a result of direct extension through the facial plane between the tonsils and the parapharyngeal space or by haematogenous or

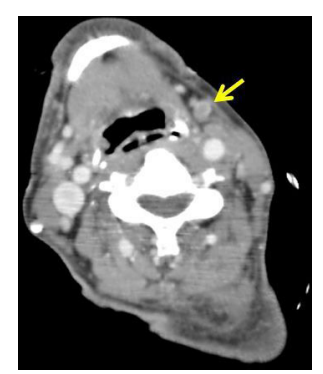

Figure 1 Axial section of $\mathrm{CT}$ of the neck showing absence of contrast in the left internal jugular vein (arrow) indicating thrombosis of the left internal jugular vein.

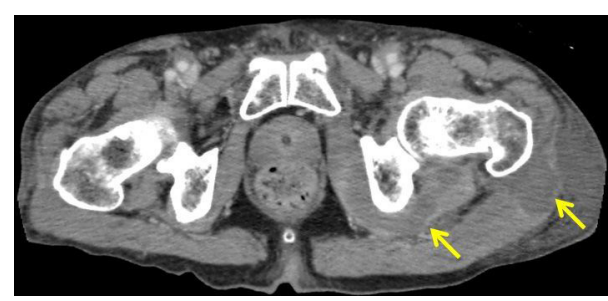

Figure 2 Contrast CT scan of the abscesses in the gluteal muscles.

lymphatic spread from the peritonsillar vessels. ${ }^{2}$ G. adiacens is a type of nutritionally variant streptococcus found as part of the normal microbiota in the oral cavity and is an uncommon source of infection. ${ }^{3}$ They are found in dental plaque and can cause endodontic infection and dental abscesses. However, they can also cause more serious bloodstream infections including endocarditis or device infections. ${ }^{4}$ This is the first reported case of IJV thrombophlebitis associated with G. adiacens bacteraemia. It is usual to have haematogenous spread to other organs from IJV thrombophlebitis, and the most common metastatic target is the lungs. ${ }^{2}$ In addition, metastasis of soft tissues has been reported, including bilateral psoas abscesses. ${ }^{5}$ In our case, the pulmonary embolism and gluteal muscle abscesses could be considered as metastasis from IJV thrombosis.

Empirical treatment for jugular vein suppurative thrombophlebitis should include a beta-lactam with a beta-lactamase inhibitor and an antibiotic with good anaerobic coverage. ${ }^{6}$ In a recent literature review of antibiotic treatment of IJV thrombophlebitis, suggested empirical antibiotic options included ampicillin-sulbactam, penicillin plus metronidazole, clindamycin or imipenem.

There are no established recommendations for the duration of antibiotic therapy because the clinical course of IJV thrombophlebitis is variable. Deep secondary abscesses are often slow to respond to antibiotic therapy because of difficulty of antibiotic penetration. Antibiotic therapy is usually administered for 3-6 weeks, although the duration of treatment can vary from 9 to 128 days. ${ }^{1}$ In our case, microbiologists recommended antibiotics to be administered for an additional 2 weeks at discharge, because the result of an MRI on day 90 showed gluteal abscesses had not completely resolved. Consequently, the total duration of antibiotic therapy was 112 days.

The role and optimal duration of anticoagulation for jugular vein suppurative thrombophlebitis remains controversial. It has been argued that jugular vein thrombophlebitis will spontaneously resolve, and many have reported successful treatment in patients without anticoagulation. ${ }^{8-11}$ Therefore, we commenced anticoagulant therapy only after the CT scan on day 40 showed that the thrombus in the left cervical vein remained unchanged. Considering that the bedridden state and immobilisation of our

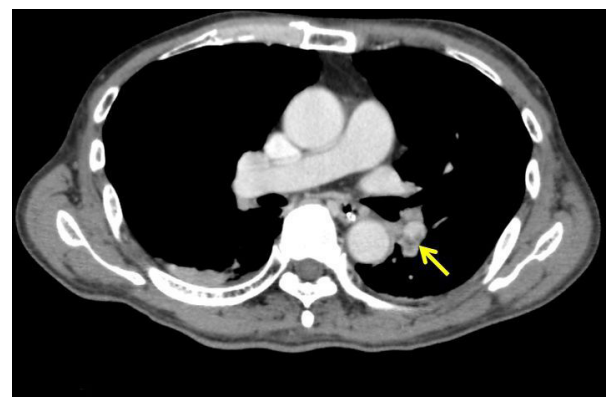

Figure 3 Contrast CT scan showing pulmonary embolism. 
patient would last for years due to his psychiatric problem, we decided to continue oral anticoagulants to prevent deep venous thrombosis.

Although Fusobacterium spp are responsible for the majority of cases of jugular vein thrombophlebitis, other members of the human oral cavity flora of the Streptococcus spp including $S$. pyogenes, S. intermedius and S. constellatus have been reported to be causative organisms. ${ }^{12-14}$ In a reported case of IJV thrombosis caused by $S$. intermedius, only a history of gingival scraping that the patient underwent 2 weeks before the onset of severe neck pain was considered to be the causative link, with the patient exhibiting a normal oropharyngeal examination and no active gingivitis. ${ }^{13}$ In our case, apart from periodontitis, no apparent primary infection site was detected in the head and neck region by physical examination and imaging. Although we did not isolate and confirm the presence of G. adiacens in the oral cavity of our patient, the anatomical proximity between the IJV and the periodontitis which might be associated with G. adiacens could be the main factor for development of IJV thrombosis in this case.

In addition, Fusobacterium spp are known to cause thromboembolic events possibly due to endotoxins that promote platelet aggregation. ${ }^{15}$ On the other hand, endovascular infection by $G$. adiacens is associated with its fibronectin-binding capacity, an essential process for endovascular bacterial adhesion during the formation of endocardial vegetations. ${ }^{16}{ }^{17}$ Although thromboembolism has not been reported with G. adiacens, its strong endovascular binding properties might contribute to the formation of IJV thrombophlebitis. However, further reports of thrombophlebitis associated with G. adiacens are needed to confirm its potential to cause venous thrombosis.

\section{Learning points}

- Granulicatella adiacens is part of the oral microflora and an uncommon cause of infection; however, it can cause serious bloodstream infections.

- Even though Fusobacterium spp are the most common cause of septic thrombophlebitis of the IJV, other oral bacteria have also been associated with this condition.

- In cases of bacteraemia caused by organisms which are part of the oral flora in patients with poor oral hygiene, the possibility of IJV thrombophlebitis deserves to be taken into consideration as a rare complication.
We experienced a devastating case of IJV thrombophlebitis associated with $G$. adiacens bacteraemia and accompanied by pulmonary embolism and intramuscular gluteal abscesses. To the best of our knowledge, there have been no reports of IJV thrombosis associated with G. adiacens. In cases of bacteraemia caused by oral microflora, we need to be aware of the fact that these organisms can cause IJV thrombophlebitis with complications of multiple metastases to other organs.

Contributors HK: Drafting the manuscript. TS: Critical revision of the manuscript.

Funding The authors have not declared a specific grant for this research from any funding agency in the public, commercial or not-for-profit sectors.

Competing interests None declared.

Patient consent for publication Next of kin consent obtained.

Provenance and peer review Not commissioned; externally peer reviewed.

\section{REFERENCES}

1 Syed Ml, Baring D, Addidle M, et al. Lemierre syndrome: two cases and a review. Laryngoscope 2007;117:1605-10.

2 Chirinos JA, Lichtstein DM, Garcia J, et al. The evolution of Lemierre syndrome: report of 2 cases and review of the literature. Medicine 2002;81:458-65.

3 Karched M, Bhardwaj RG, Tiss A, et al. Proteomic analysis and virulence assessment of Granulicatella adiacens secretome. Front Cell Infect Microbiol 2019:9:104.

4 Cargill JS, Scott KS, Gascoyne-Binzi D, et al. Granulicatella infection: diagnosis and management. J Med Microbio/ 2012:61:755-61.

5 Bird NT, Cocker D, Cullis P, et al. Lemierre's disease: a case with bilateral iliopsoas abscesses and a literature review. World J Emerg Surg 2014;9:38.

6 Wong AP, Duggins ML, Neil T. Internal jugular vein septic thrombophlebitis (Lemierre syndrome) as a complication of pharyngitis. J Am Board Fam Med 2015;28:425-30.

7 Eilbert W, Singla N. Lemierre's syndrome. Int J Emerg Med 2013;6:40.

8 Phua CK, Chadachan VM, Acharya R. Lemierre syndrome-should we anticoagulate? A case report and review of the literature. Int J Angio/ 2013;22:137-42.

9 Lu MD, Vasavada Z, Tanner C. Lemierre syndrome following oropharyngeal infection: a case series. J Am Board Fam Med 2009:22:79-83.

10 Dool H, Soetekouw R, van Zanten M, et al. Lemierre's syndrome: three cases and a review. Eur Arch Otorhinolaryngol 2005:262:651-4.

11 Lee W-S, Wang F-D, Shieh Y-H, et al. Lemierre syndrome complicating multiple brain abscesses caused by extended-spectrum $\beta$-lactamase-producing Klebsiella pneumoniae cured by fosfomycin and meropenem combination therapy. J Microbiol Immunol Infect 2012:45:72-4.

12 Anton E. Lemierre syndrome caused by Streptococcus pyogenes in an elderly man. Lancet Infect Dis 2007;7:233.

13 Gupta S, Merchant SS. Lemierre's syndrome: rare, but life threatening - a case report with Streptococcus intermedius. Case Rep Med 2012;2012:1-4.

14 Shimada M, Morinaga Y, Kitazaki T, et al. A severe case of Lemierre syndrome with Streptococcus constellatus infection. Jpn J Infect Dis 2014;67:488-9.

15 Laurencet M-E, Rosset-Zufferey S, Schrenzel J. Atypical presentation of Lemierre's syndrome: case report and literature review. BMC Infect Dis 2019;19:868.

16 Patil SM, Arora N, Nilsson P, et al. Native valve infective endocarditis with osteomyelitis and brain abscess caused by Granulicatella adiacens with literature review. Case Rep Infect Dis 2019;2019:1-6.

17 Yamaguchi T, Soutome S, Oho T. Identification and characterization of a fibronectinbinding protein from Granulicatella adiacens. Mol Oral Microbiol 2011;26:353-64.

Copyright 2021 BMJ Publishing Group. All rights reserved. For permission to reuse any of this content visit

https://www.bmj.com/company/products-services/rights-and-licensing/permissions/

BMJ Case Report Fellows may re-use this article for personal use and teaching without any further permission.

Become a Fellow of BMJ Case Reports today and you can:

- Submit as many cases as you like

- Enjoy fast sympathetic peer review and rapid publication of accepted articles

- Access all the published articles

Re-use any of the published material for personal use and teaching without further permission

Customer Service

If you have any further queries about your subscription, please contact our customer services team on +44 (0) 2071111105 or via email at support@bmj.com.

Visit casereports.bmj.com for more articles like this and to become a Fellow 